as myocardial segments. No difference in progression rate was observed stratifying patients according to disease subset or other clinical parameters.

Conclusion: GLS impairment progressed over a 20-month follow-up period in a cohort of SSc patients without clinically overt cardiac involvement. Further studies are needed to assess the significance of subclinical heart involvement and its progression in patients with SSc.

Disclosure of Interests: : None declared

DOI: 10.1136/annrheumdis-2020-eular.2824

\begin{tabular}{|l|l}
\hline SAT0308 & SCREENING TOOLS FOR PULMONARY ARTERIAL \\
HYPERTENSION (PAH) IN SYSTEMIC SCLEROSIS \\
(SSC): A SYSTEMATIC LITERATURE REVIEW (SLR)
\end{tabular}

C. Bruni ${ }^{1}$, G. De Luca ${ }^{2,3}$, M. G. Lazzaroni ${ }^{4}$, E. Zanatta ${ }^{5}$, G. Lepri ${ }^{1}$, M. MatucciCerinic ${ }^{1,6} .{ }^{1}$ University of Florence, Faculty of Medicine, Experimental and Clinical Medicine, Division of Rheumatology, Firenze, Italy; ${ }^{2}$ IRCCS San Raffaele Hospital, Immunology, Rheumatology, Allergology and Rare diseases, Milan, Italy; ${ }^{3}$ Vita-Salute San Raffaele University, Milano, Italy; ${ }^{4}$ ASST Civil Hospital of Brescia, Rheumatology and Clinical Immunology, Brescia, Italy; ${ }^{5}$ University of Padua, Medicine, Rheumatology Unit, Padova, Italy; ${ }^{6}$ Careggi University Hospital, Rheumatology, Firenze, Italy

Background: in SSc, PAH has a high morbidity and mortality burden. Therefore, screening and early detection are pivotal to achieve an early diagnosis of $\mathrm{PAH}$. Objectives: to search the literature for all screening modalities for SSc-PAH in reference to right heart catheterization as diagnostic gold standard.

Methods: papers from 2 previously published SLRs [22 from Glaude et al (1) - from inception to 19/06/2012 - and 22 from Young et al 2018 - from 20/06/2012 to $02 / 10 / 2017$ ] were included. The articles' database was integrated with a systematic search on Pubmed, EMBASE, Web of Science for papers published from $03 / 10 / 2017$ to $31 / 12 / 2018$. A total of 199 papers were reviewed and 32 were finally extracted. Bias risk was assessed through QUADAS2 tool.

Results: 167 papers were excluded from data extraction mainly for $\mathrm{PAH}$ screening non as main focus or for non-including SSc patients. The 32 papers extracted presented a low bias risk according to QUADAS2. Screening methods reported were:

- Echocardiographic parameters in 31/32 studies, in particular systolic pulmonary arterial pressure (sPAP) in 22 papers; $40 \mathrm{mmHg}$ was the most frequently used cut-off (in 12/22 papers); SPAP was part of a composite algorithm in 9/22 papers. Among others, tricuspid regurgitation velocity (TRV) was used in 6/31 (as part of composite 5/6) and right atrial pressure (RAP) in 3/31 papers.

- Pulmonary function tests parameters in 22/32 papers, with \% predicted Lung diffusion for carbon oxyde (DLco) in 21 papers, with a 50\% cut-off in 11/21 and as part of composite algorithm in 13/21 studies. Moreover, walked distance at six minutes walking test was a screening parameter in 3/32 papers.

- Serum biomarkers in 12/32 papers, with anti-centromere antibodies (6/12), NT-proBNP (6/12) and uric acid (5/12) being the most frequently reported.

- Clinical parameters in 15/32 papers, with unexplained dyspnoea in 9/15 and telangiectasias in $5 / 15$ papers.

- Composite algorithms were used in 18/32 manuscripts: among them, DETECT (5/18), ESC/ERS 2009 (4/18) or 2015 (3/18) guidelines, ASIG (2/18) e ITINERair (1/18). In different cohorts, DETECT and ASIG showed higher sensitivity and negative predictive value than ESC/ERS 2009
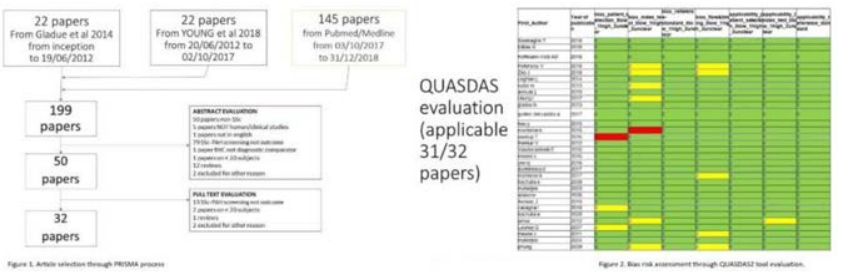

Conclusion: in the literature, the screening of SSc-PAH is largely investigated by echocardiographic parameters. In particular, SPAP and TRV, both as single items or part of a composite algorithm, including also serum biomarkers, clinical and functional parameters, are the most frequent parameters evaluated.

(supported by an Actelion Pharmaceuticals unrestricted research grant)

References:

[1] Gladue $\mathrm{H}$, et al. Screening and diagnostic modalities for connective tissue disease-associated pulmonary arterial hypertension: a systematic review. Semin Arthritis Rheum. 2014 Feb;43(4):536-41.
[2] Young A, et al. Update of screening and diagnostic modalities for connective tissue disease-associated pulmonary arterial hypertension. Semin Arthritis Rheum. 2019 Jun;48(6):1059-1067.

Acknowledgments: (supported by an Actelion Pharmaceuticals unrestricted research grant)

Disclosure of Interests: Cosimo Bruni Speakers bureau: Actelion, Eli Lilly, Giacomo De Luca Speakers bureau: SOBI, Novartis, Celgene, Pfizer, MSD, Maria Grazia Lazzaroni: None declared, Elisabetta Zanatta: None declared, Gemma Lepri: None declared, Marco Matucci-Cerinic Grant/research support from: Actelion, MSD, Bristol-Myers Squibb, Speakers bureau: Acetelion, Lilly, Boehringer Ingelheim

DOI: 10.1136/annrheumdis-2020-eular.1953

\section{SAT0309 \\ CARDIAC MAGNETIC RESONANCE IMAGING ELEVATED NATIVE MYOCARDIAL T1 IS PREDICTIVE FOR THE DEVELOPMENT OF MYOCARDIAL DYSFUNCTION IN SYSTEMIC SCLEROSIS}

E. Chatelus ${ }^{1}$, V. Poindron ${ }^{1}$, M. Canuet ${ }^{2}$, A. S. Korganow ${ }^{1}$, P. Germain ${ }^{3}$, T. Martin ${ }^{1}$, S. El Ghannudi ${ }^{3} .{ }^{1}$ National Reference Center For Autoimmune Diseases RESO, Hôpitaux Universitaires de Strasbourg, Strasbourg, France; ${ }^{2}$ Pneumology Department, Hôpitaux Universitaires de Strasbourg, Strasbourg, France; ${ }^{3}$ Radiology Department, Hôpitaux Universitaires de Strasbourg, Strasbourg, France

Background: All patients included in the study fulfilled the ACR/EULAR classification criteria for SSc. We prospectively included patients who underwent at least two CMR at 1.5T, including native T1 and T2 mapping (which give account for myocardial fibrosis and myocardial edema respectively), left and right ventricles morphology and functional assessment, and Late Gadolinium Enhancement (LGE) as a part of routine follow-up between 2015 and 2019.

Objectives: To evaluate the prognostic value of initial abnormal T1 mapping. Methods: All patients included in the study fulfilled the ACR/EULAR classification criteria for SSc. We prospectively included patients who underwent at least two CMR at 1.5T, including native T1 and T2 mapping (which give account for myocardial fibrosis and myocardial edema respectively), left and right ventricles morphology and functional assessment, and Late Gadolinium Enhancement (LGE) as a part of routine follow-up between 2015 and 2019.

Results: Sixty-three patients underwent at list two CMR during the study period. Forty-three patients were women. Mean age was $52.5 \pm 15.5$ years old. Follow-up duration between the initial and the follow-up CMR was $14.5 \pm 11.5$ months. Forty-one had diffuse SSc. The mean native T1 was $1066.8 \pm 44.6 \mathrm{~ms}$. Twenty-one patients suffered from cardiac clinical manifestations. Nine patients died during the follow-up. Thirty patients $(47.6 \%)$ had elevated T1 (ET1) with mean T1 $1105.4 \pm 36.7 \mathrm{~ms}$ at the time of initial CMR. Initial ET1 was clearly correlated with: $1 /$ alteration of Left Ventricle (LV) Ejection fraction (EF) $(r=0.5, p<0.0001)$ during the study period, $2 / \mathrm{LV}$ dilatation at initial screening and follow up $(r=0.22, p=0.03$ and $r=0.3, p=0.02$ ). Regarding Right ventricle, initial ET1 was correlated with initial Right Ventricle $(\mathrm{RV})$ dilatation $(r=0.3, p=0.02)$ but neither with $R V$ volume nor RVEF at follow-up. Interestingly, initial ET1 correlated with pericardial effusion $(r=0.3, p=0.003$ ) which is known to be a pejorative prognostic factor. Seventeen patients (28\%) had LGE but the ET1 at initial screening and follow-up was not correlated with LGE.

Six patients had elevated T2 (ET2) which correlated with initial and follow up LV dilatation $(r=0,32, p=0.002$ and $r=0.5, p<0.0001$ respectively) but not with LVEF during the period study. Among other parameters, initial increased BNP was correlated with follow up ET1 LVEF and RVEF $(r=0.4, p=0.01 ; r=0.35, p=0.007$; $r=0,37, p=0.005$ respectively). In the same way, initial Pulmonary Arterial Hypertension $(\mathrm{PAH})$ was correlated with follow up ET1 $(r=0.3, p=0.02)$. Initial ET1 did not correlate with age, sex, cardiovascular risk factors, cardiac manifestations or death.

Conclusion: Assessment of diffuse myocardial fibrosis by native $\mathrm{T} 1$ is predictive of the occurrence of cardiac dysfunction at the follow-up as initial ET1 was associated with decreased left ventricular function and LV and RV dilatations). These data highlights the potential role of CMR with T1mapping in initial screening and at the follow-up and provides new insights in the cardiac SSc follow up strategy.

\section{References:}

[1] Poindron V, Chatelus E, Canuet M, Gottenberg JE, Arnaud L, Gangi A Gavand PE, Guffroy A, Korganow AS, Germain P, Sibilia J, El Ghannudi S, Martin T. T1 mapping cardiac magnetic resonance imaging frequently detects subclinical diffuse myocardial fibrosis in systemic sclerosis patients. Semin Arthritis Rheum. 2019 Jun 19.

Disclosure of Interests: None declared

DOI: 10.1136/annrheumdis-2020-eular.5492 